\title{
Aquiles, la Tortuga y la aplicación de normas jurídicas como un "asunto particularista" sin particularismo
}

\author{
Achilles, the Tortoise and the Application of Legal Rules \\ as a "Particularistic Affair" without Particularism
}

María Gabriela Scataglini*

Resumen: Me concentro en dos aspectos del artículo de Duarte d'Almeida “¿Qué es aplicar el derecho?” que identifico como: 1) que el silogismo jurídico es una mala reconstrucción del razonamiento que conduce $a-y$ a la vez justifica- una sentencia judicial; y 2) que la inferencia que lleva a una decisión judicial es de carácter particularista, no habiendo ningún principio universal con el cual los jueces se comprometan al aplicar una norma a un caso. Respecto de 1) trazo cierta analogía con el diálogo de Lewis Carroll "Lo que la Tortuga le dijo a Aquiles" y recurro a la concepción de Gilbert Ryle acerca de la inferencia como un saber cómo. Basada en las consideraciones ryleanas expongo el alcance de mi coincidencia con 2), y por último planteo una discrepancia acerca de qué considerar incluido en lo que el autor llama "aplicación pragmática" del derecho.

Palabras clave: silogismo jurídico, inferencia, saber cómo, particularismo, aplicación pragmática.

Doctora de la Universidad de Buenos Aires. Profesora adjunta interina de Teoría General del Derecho de la Universidad de Buenos Aires, Argentina. Correo electrónico: g_scataglini@hotmail.com 


\begin{abstract}
I focus on two aspects of Duarte d'Almeida's paper "What is it to apply law?" identified as: 1) the legal syllogism is a bad reconstruction of the reasoning that leads to - and also justifies - a judicial decision; and 2) that the inference leading to a judicial decision is of a particularistic nature, not being an universal principle with which judges commit themselves when applying a legal rule. Regarding 1) I show some analogy with Lewis Carroll's dialogue "What the Tortoise said to Achilles" and draw upon Gilbert Ryle's conception of inference as a know how. Based on Rylean considerations, I mark the scope of my agreement on 2), and finally I disagree on what is to be involved in what the author calls "pragmatic application" of law.
\end{abstract}

Keywords: legal syllogism, inference, know how, particularism, pragmatic application.

\title{
Introducción
}

En “¿Qué es aplicar el derecho?”, Luís Duarte d’Almeida presenta una serie de interesantes reflexiones acerca de en qué consiste aplicar normas jurídicas. Una de sus consideraciones - en la que aquí me voy a centrar-constituye un desafío a una de las ideas más arraigadas de la teoría jurídica: la de que la decisión judicial puede ser reconstruida mediante un razonamiento silogístico cuya primera premisa es la norma jurídica, la segunda premisa una descripción de los hechos del caso, y la conclusión la sentencia del juez que resuelve el caso. A esta reconstrucción suele aludirse como "silogismo jurídico" o "silogismo judicial".

A mi modo de ver pueden distinguirse dos aspectos en la crítica de Duarte d’Almeida al silogismo jurídico: el primero de ellos sería que este, tal como usualmente se lo concibe (del modo descripto en el párrafo anterior), está mal construido: se trata, según entiendo, de un argumento acerca de cómo reconstruir adecuadamente el razonamiento lógico que conduce a - y a la vez justifica- la resolución judicial de un caso en base a una norma jurídica. Duarte d’Almeida hace una propuesta alternativa acerca de cuáles deberían ser las premisas de esa reconstrucción. El segundo aspecto de la crítica, me parece, alude a una cuestión sustantiva: la inferencia que conduce a una decisión judicial es de carácter eminentemente particularista; 
Aquiles, la Tortuga y la aplicación de normas jurídicas como un "asunto...

aquí la tesis central es que no hay ningún principio universal con el cual los jueces se comprometan al aplicar una norma: al resolver un caso los jueces no determinan cuándo (en qué casos en general) esa norma resulta aplicable, sino sencillamente que la norma en cuestión se aplica a ese caso que él o ella tienen que resolver.

Aún a riesgo de simplificar demasiado, trataré de presentar los planteos de Duarte d’Almeida del modo más sencillo posible, y al tiempo expondré mis coincidencias, discrepancias o cuestionamientos en torno a ellos. Otra cuestión importante a dilucidar será si, entre los dos aspectos que he identificado, existe alguna relación o se trata de dos críticas conceptualmente independientes entre sí.

\section{El “silogismo jurídico" está mal construido}

Esta parece ser la conclusión de Duarte d'Almeida, tras un minucioso análisis en base al cual afirma, básicamente, que si se toma como primera premisa la norma jurídica aplicable, la conclusión del razonamiento no resulta ser la decisión judicial (sentencia). Para poder derivar lógicamente un enunciado que constituya una sentencia o decisión judicial, se requeriría un razonamiento tal que: i) la primera premisa fuera un enunciado hipotético cuyo antecedente contuviera una cláusula condicional del tipo "si la norma en cuestión se aplica a este caso" y el consecuente fuera el contenido de la sentencia; ii) la segunda premisa fuera la afirmación de que la norma en cuestión se aplica a ese caso; y entonces sí, a partir de esas dos premisas se deduciría la sentencia por Modus Ponens. ${ }^{1}$

1 Transcribiendo el ejemplo de Duarte d'Almeida (2021, Sección 2.4) un razonamiento así tiene la siguiente forma:

“(1’) Si el párrafo 8 del artículo III de las Reglas de La Haya-Visby se aplica a la cláusula 2 del conocimiento de embarque expedido por los demandados, entonces la cláusula 2 del conocimiento de embarque expedido por los demandados es nula y sin efecto.

(2’) El párrafo 8 del artículo III de las Reglas de La Haya-Visby se aplica a la cláusula 2 del conocimiento de embarque expedido por los demandados.

Por lo tanto, (de (1') y (2')),

(3') La cláusula 2 del conocimiento de embarque emitido por los acusados es nula y sin efecto". 
Lo que señala Duarte d'Almeida respecto del modelo que propone es: i) que la primera premisa no es la norma que está siendo aplicada (como en el modelo del silogismo jurídico), y ii) que la segunda premisa es un enunciado de segundo orden, es decir, que habla sobre la aplicabilidad de la norma a los hechos del caso - i. e. afirmación de aplicabilidad-, y no una descripción de los hechos del caso (como en el modelo del silogismo jurídico).

Con ello quedaría demostrado que el modelo del silogismo jurídico es inapropiado para reconstruir lo que pretende, a saber, un razonamiento cuya conclusión es la decisión judicial (sentencia).

En un artículo precedente al aquí comentado, Duarte d’Almeida señala:

Rule-deductivists, by contrast, seem to want to cash out in first-order terms the second-order idea that a statutory rule applies to a case. But how could that be right? If the justification of the court's decision-the justification of the court's applying a provision to the case in hand-turned, as rule-deductivists claim, on the fact that the case satisfies the antecedent of the corresponding statutory rule, then what we would need as a premise in the argument would be the second-order statement that the antecedent of the rule is satisfied by the case-not a statement of the relevant rule together with a statement of facts that satisfy its antecedent. Conversely, an argument that featured a first-order statement of the relevant rule as a premise, and combined it, as in the rule-deductivists' legal syllogism, with a description of facts that satisfy the antecedent of the rule, could not itself justify the second-order claim that the rule applies to the facts (Duarte d'Almeida 2019, p. 34).

La crítica de Duarte d'Almeida — si bien podría tildársela de minuciosa- resulta desafiante. Siguiendo su línea de pensamiento, parece correcto que el silogismo jurídico no sería una reconstrucción rigurosa de aquello en lo que consiste "aplicar una norma a un caso", en el sentido de que, tal como él muestra, de premisas de primer orden (la norma y la descripción de los hechos) no se seguiría como conclusión el resultado de aplicar la norma al caso (que es el contenido de la sentencia judicial). Pero si Duarte d'Almeida está en lo correcto, ¿ cómo es que esto ha pasado hasta ahora inadvertido? 
Aquiles, la Tortuga y la aplicación de normas jurídicas como un "asunto...

No ha sido así. Que las premisas de un razonamiento de primer orden no "conducen" necesariamente a la conclusión ya había sido célebremente señalado.

En efecto, hay algún aspecto de la crítica de Duarte d'Almeida al silogismo jurídico que podría relacionarse con el argumento de Lewis Carroll en su artículo "Lo que la Tortuga dijo a Aquiles" (Carroll, 1895).

Como es sabido, en el diálogo imaginario de Carroll, la Tortuga le pide a Aquiles que anote en su libreta los siguientes enunciados:

(A) Dos cosas que son iguales a una tercera son iguales entre sí.

(B) Los dos lados de este triángulo son iguales a un tercero.

(Z) Los dos lados de este triángulo son iguales entre sí.

La Tortuga pregunta a Aquiles si la conclusión se deduce lógicamente de las premisas, y Aquiles responde afirmativamente. Luego de algún intercambio, la Tortuga le pregunta si podría haber algún lector que dijera "Acepto A y B como verdaderas", pero se negara a aceptar el enunciado condicional que dice que "si A y B son verdaderas, entonces $\mathrm{Z}$ debe ser verdadera" - llamemos a ese enunciado hipotético " $\mathrm{C}$ " - . Entonces, la posición de la Tortuga es que acepta A y B pero no acepta C. Y le pide a Aquiles que la fuerce a aceptar $Z$. Aquiles se da cuenta de que para poder hacer que la Tortuga acepte Z, ella debería aceptar C. Entonces, la Tortuga le dice que solo aceptará C si Aquiles la anota en su libreta. Ahora Aquiles insiste en que, si A, B y C son verdaderas, la Tortuga debe aceptar Z.

"Si A y B y C son verdaderas, $\mathrm{Z}$ debe ser verdadera", repitió pensativamente la Tortuga. “ ¿Esa es otra Hipótesis, o no? Y, si no reconociera su veracidad, ¿podría aceptar A y B y C, y todavía no aceptar Z, o no?"

Y entonces la Tortuga le pide a Aquiles que anote esa otra hipótesis - llamémosla (D) - en su libreta. El cuento termina relatando que varios meses después, Aquiles seguía anotando enunciados hipotéticos en su libreta y la Tortuga aún no aceptaba Z .

El problema es el del regreso al infinito: si para aceptar una conclusión lógica debemos aceptar la hipótesis de que si las premisas son verdaderas la conclusión también lo es, y si tratamos a esa hipótesis, en tanto verdadera, 
como una premisa más, se incurre en un regreso, porque necesitaremos siempre otra hipótesis más que afirme que si esa nueva premisa es verdadera la conclusión también lo es.

La paradoja es el resultado de suponer que la tarea de Aquiles es la de proveer premisas suficientes para obligar a la Tortuga a aceptar la conclusión, y lo que sucede es que la incorporación sucesiva de hipótesis solo conduce al regreso. ¿Cuál es la enseñanza principal del relato de Carroll? La siguiente: que la hipótesis -i.e. el condicional que la Tortuga debe "aceptar" para pasar a la conclusión - no debe ser tratada como una premisa más porque constituye un enunciado de segundo orden.

Este es el punto en el que creo que es trazable alguna semejanza con el planteo de Duarte d'Almeida, quien parece partir de la misma intuición que tiene la Tortuga: que las dos premisas de primer orden no llevan - no obligan - a aceptar la conclusión. Para afirmar la conclusión se necesita hacer algo más, algo que solo puede ser captado (reconstruido) en un orden superior. Pero a su vez, el (o los) enunciados de segundo orden no pueden aparecer "mezclados" en el mismo razonamiento con las premisas de primer orden.

Ahora bien, existen diferencias relevantes. En el relato de Aquiles y la Tortuga el enunciado de segundo orden (lo que denominan la Hipotética o la hipótesis: que si $\mathrm{A}$ y $\mathrm{B}$ y $\mathrm{C}$ son verdaderas, $\mathrm{Z}$ debe serlo) refiere al pasaje de las premisas a la conclusión. En cambio, en el planteo de Duarte d'Almeida el tipo de enunciado de segundo orden es un enunciado sobre la aplicabilidad de la norma al caso. Y además se trata de dos enunciados, mejor dicho, de las dos premisas de un razonamiento; recuérdese: la hipótesis de que si una norma se aplica a un caso entonces se obtiene como conclusión el resultado de esa aplicación (i. e. la sentencia), y la afirmación — también de segundo orden- de que esa norma se aplica al caso.

Veamos esto más en detalle: los enunciados A y B del diálogo entre Aquiles y la Tortuga son de primer orden, al igual que los del silogismo jurídico. Si hiciéramos el ejercicio de trasladar la propuesta de Duarte d'Almeida al ejemplo de la tortuga, obtendríamos este razonamiento:

(A') Si (A) se aplica a este triángulo, los dos lados de este triángulo son iguales entre sí. 
Aquiles, la Tortuga y la aplicación de normas jurídicas como un "asunto...

(B') (A) se aplica a este triángulo.

(Z) Los dos lados de este triángulo son iguales entre sí.

Resulta evidente que se trata de un razonamiento de segundo orden, dado que (A) ("Dos cosas que son iguales a una tercera son iguales entre sí”) está siendo mencionada y no usada. El punto de Duarte d'Almeida residiría en que es este razonamiento - y no el de primer orden (i. e. el que aparece en el cuento) - el que lleva a la conclusión.

Ahora bien, ¿puede la moraleja del relato de Carroll arrojar alguna luz sobre la propuesta de Duarte d'Almeida? Como ya se dijo, la paradoja muestra que los enunciados de segundo orden requeridos para llegar a la conclusión no pueden formar parte del mismo razonamiento junto con las premisas de primer orden. Lo que la hipótesis expresa no puede ser afirmado junto con los otros enunciados como una premisa más. ¿Pero qué es lo que la hipótesis expresaba en el relato de Aquiles y la Tortuga? Como también se mencionó, aquella refería al pasaje de las premisas a la conclusión. Según las interpretaciones más usuales esa hipótesis constituye un principio de inferencia: los principios de inferencia son "tickets", habilitaciones o permisos para extraer conclusiones. Su aceptación justifica y permite el pasaje a la conclusión. Pero se trata de reglas (operaciones) y no de premisas (verdades). Lo que se pone en evidencia en el diálogo es que no se trata aceptar nuevas "verdades", lo que se requiere son reglas de transformación que habiliten el tránsito de premisas a conclusión. En esta línea, el análisis inaugurado por Ryle indica que la operación de pasar de las premisas a la conclusión (i. e. la aplicación de reglas inferenciales) requiere un tipo de conocimiento que no es una forma de saber que, sino de saber cómo (Ryle, 1949). ${ }^{2}$ La paradoja surge precisamente porque se malinterpreta qué quiere decir saber inferir. Inferir no puede ser reducido o analizado como el conocimiento de ciertas proposiciones. La práctica

2 Ryle explica: "It is therefore possible for people intelligently to perform some sorts of operations when they are not yet able to consider any propositions enjoining how they should be performed. Some intelligent performances are not controlled by any anterior acknowledgments of the principles applied in them" $(1949$, p. 30). 
inferencial supone saber operar con ciertas reglas, lo cual se traduce en realizar inferencias válidas. ${ }^{3}$

¿Qué resultaría de trasladar estas enseñanzas a la propuesta de Duarte d'Almeida? Aquí aquello que hay que saber cómo hacer no consiste - o no consiste solo- en pasar de las premisas a la conclusión, sino específicamente en saber si y cómo se relacionan la primera y la segunda premisa, en otras palabras, saber reconocer si la norma es aplicable al caso. Deberíamos entonces concluir que el reconocimiento de la relación de aplicabilidad es un tipo de saber cómo no reductible a la aceptación de proposiciones. ¿Pero es esto lo que Duarte d'Almeida quiere afirmar?

La respuesta no resulta clara. Como se dijo, el foco de su planteo está puesto en la relación de aplicabilidad. Su razonamiento de segundo orden afirma - en su segunda premisa - que existe esa relación entre la norma y aquello a lo que se aplica. Esto parece equiparable a la idea de subsunción. Aplicar una norma jurídica a un objeto es aceptar que ese objeto se "subsume" en la norma.

Pero la cuestión resulta confusa en algunos aspectos. En primer lugar, el ejemplo de Duarte d'Almeida es un poco desorientador porque alude a la aplicabilidad de la norma a un "objeto", que en el caso consiste, efectivamente, en una cláusula contractual: el resultado de esa aplicación es tornar nula la cláusula. Pero no queda claro cómo estas características particulares del ejemplo se traducen a y/o si alcanzan a otras normas en las cuales lo que se tiene como "objeto de la subsunción" no es propiamente un objeto sino un hecho (como ser, por ejemplo, una acción llevada a cabo por una persona). La insistencia de Duarte d'Almeida en hablar de "objeto" y los términos en que lo hace torna dudosa esta cuestión.

3 Peter Winch retoma esta línea y la profundiza: "the actual process of drawing an inference, which is after all at the heart of logic, is something which cannot be represented as a logical formula (...) Learning to infer is not just a matter of being taught about explicit logical relations between propositions; it is learning to do something" (1958, p. 57). Winch llega a sugerir que la moraleja del diálogo de Carroll es un caso particular de una lección general, en el sentido de que la correcta aplicación de las normas que rigen una forma de actividad humana no puede ser resumida en una serie de otras normas, o en un conjunto de preceptos explícitos. 
Aquiles, la Tortuga y la aplicación de normas jurídicas como un "asunto...

Por otro lado, el problema planteado por Duarte d'Almeida parece limitarse exclusivamente a la actividad de aplicar normas jurídicas para resolver un caso en el ámbito del derecho, y excluir la aplicación de reglas en general. ${ }^{4}$ Sin embargo, el argumento de Carroll sí alcanza a todo razonamiento en base a reglas; y en general a cualquier razonamiento deductivo. Aun a los de segundo orden.

Siendo esto así, la paradoja también alcanza al razonamiento sobre la aplicabilidad que presenta Duarte d'Almeida como alternativa al silogismo jurídico. Véase ello en mi esquema precedente de razonamiento de segundo orden: según la paradoja, el pasaje de (A') y (B') a la conclusión (Z’) solo resultaría justificado - nos veríamos obligados a dar ese paso- si se acepta otro enunciado, diríamos de tercer orden, y así sucesivamente. Un modo de escapar al regreso es asumir la línea de Ryle: todo argumento deductivo, aun uno de segundo orden, padece la paradoja a menos que inferir sea concebido como un saber práctico irreductible a proposiciones. Pero ¿aceptaría esto Duarte d’Almeida? ¿O quizás él considera que por ser sobre aplicabilidad (digamos, un segundo orden de tipo diferente al del relato de Carroll que era sobre la inferencia) su propuesta de reconstrucción —que es obviamente deductiva- puede escapar al regreso en razón de algún otro argumento? ¿Quizás alguno relacionado con el hecho de que su primera premisa no tiene la forma de un enunciado universal al modo de "Para todo x..."?

Si así fuera, resultaría interesante conocer sus argumentos, porque podrían relacionarse con lo que he identificado como el segundo aspecto de su crítica, relativa al carácter particularista de los razonamientos jurídicos. Pero en tal caso, no alcanzo a ver, todavía, cuál sería exactamente el vínculo entre ambos aspectos. En otras palabras, ¿cómo se relaciona - si es que lo hace- la crítica al silogismo jurídico como una mala reconstrucción, con aquella otra relativa al carácter particularista de los razonamientos que llevan adelante los jueces y juezas al resolver casos? La respuesta a esa cuestión no surge de manera nítida del trabajo de Duarte d’Almeida.

4 Tengo en cuenta que aquello a lo que Duarte d'Almeida se refiere como "aplicación pragmática” sí es propia de la práctica jurídica. Me referiré a ella en la Sección 3. Pero los argumentos que ahora estoy discutiendo son presentados por Duarte d'Almeida como característicos de lo que llama "aplicación inferencial" del derecho, y es a este tópico al que me refiero en el texto principal. 


\section{El carácter particularista de la aplicación de normas jurídicas}

Duarte d'Almeida afirma que al resolver un caso, un tribunal no se compromete con ningún enunciado reconstruible bajo una forma de este tipo: "Para todo $\mathrm{x}$, si Rx entonces $\mathrm{Nx}$ ", que es como usualmente se "traducen" las normas generales —enunciados de primer orden - al lenguaje simbólico, mostrando que se trata de enunciados universales.

$\mathrm{Al}$ respecto el autor afirma:

La aplicación del derecho es, en cierto sentido, un asunto particularista. Lo que quiero decir no es que, para cualquier conclusión correctamente alcanzada sobre la base de que alguna disposición $p$ se aplica a algún objeto $x$, no exista un principio universal verdadero que capte todos y sólo aquellos aspectos de $x$ que son relevantes para esa conclusión - un principio que podría utilizarse para construir una inferencia que se ajustara a un esquema idéntico al del silogismo jurídico. Lo que quiero decir es que nosotros- y los tribunales en particular- no nos comprometemos (de hecho, ni siquiera podría esperarse que lo hiciéramos) con formulaciones de principios de ese tipo como un paso necesario, típico o incluso mínimamente frecuente en nuestro razonamiento o en nuestros argumentos hacia tales conclusiones (Duarte d’Almeida 2021, Sección 2.4).

Aclarando cuál es el alcance de su tesis, el autor señala que su propuesta de reconstrucción se refiere enteramente a la relación entre la disposición y un objeto particular. No se trata de un argumento sobre las cláusulas (en plural) de los conocimientos de embarque (en general), ni tampoco sobre la gama de efectos que el párrafo 8 del artículo III del Reglamento podría tener sobre una cláusula de ese tipo. Se trata de un argumento sobre una determinada cláusula - la cláusula 2 del conocimiento de embarque que

5 Este esquema podría responder al ejemplo del Duarte d’Almeida abreviado: "Para toda cláusula contractual sobre conocimientos de embarque, si restringe la responsabilidad, entonces es nula". 
Aquiles, la Tortuga y la aplicación de normas jurídicas como un "asunto...

Denning tenía ante sí- y sobre el efecto de la disposición sobre ella, afirma Duarte d'Almeida (2021, Sección 2.4).

En "On the legal syllogism", aclara que no niega el carácter universalizable de la aplicación de normas jurídicas, en el sentido de que, racionalmente, el juez se compromete con que cualquier otro caso que fuera similar en todos los aspectos relevantes debería ser uno al cual la norma también resultaría aplicable. Pero señala: "My point is that his decision did not commit him to any precise and definitive view on what exactly those relevant respects were" (Duarte d'Almeida 2019, pp. 28-29) (cursiva del original).

$\mathrm{Y}$ en el trabajo en este volumen:

Cuando abordan la cuestión de si una disposición se aplica al caso que se tiene enfrente, los tribunales no formulan $-\mathrm{y}$ probablemente ni siquiera serían capaces de hacerlo- descripciones rigurosas de las propiedades que tendría que satisfacer cualquier caso al cual sea aplicable la disposición. Ni siquiera es razonable esperar que los tribunales formulen descripciones rigurosas de las propiedades relevantes del caso particular que estén tratando. No articulan ni se comprometen, como tampoco lo hace Denning, con enunciados de primer orden de las "reglas" que entienden que la disposición relevante expresa, y bajo la cual simplemente subsumirían el caso que están tratando.

Coincido con este aspecto. En otro lugar he planteado que captar "el alcance" de una norma (i. e. saber aplicarla) no puede consistir en representarse una proposición general conteniendo todas sus aplicaciones; una proposición tal que incluyera, por ejemplo, un listado de todas las excepciones posibles.

El problema no es (o no es solo) el tiempo que insumiría dar una formulación como esa. Como ha señalado Robert Brandom,

(..) no es sólo que necesitaríamos excluir una lista infinita de condiciones - aunque eso es cierto-. Es que no se podría definir la pertenencia a esa lista: no sabemos cómo especificar de antemano lo que pertenece a la lista (Brandom, 2000 [2002], p. 109). 
Lo que creo que está en juego aquí es cierta idea de lo que significa subsumir. ${ }^{6}$ Reconocer cuándo un caso particular es una instancia de un universal -i. e. la norma jurídica- no requiere tener previamente desplegado - sea mediante formulación en el lenguaje o como captación o representación mental - un listado total de circunstancias de aplicación, entre las cuales "encontrar" la que se tiene bajo análisis.

Por ello coincido con la idea de Duarte d'Almeida de que aplicar normas jurídicas no consiste en la representación ni el compromiso por parte del agente judicial de una proposición universal conteniendo todas las aplicaciones de la norma.

Es que concebir una proposición universal de esas características resulta problemático: si quisiéramos definirla por extensión, tendríamos el problema señalado por Brandom en la cita supra: además de no poder realizar una lista exhaustiva, no sabríamos qué poner en ella. Si quisiéramos definirla intensionalmente, estaríamos formulando una nueva regla; ahora bien, esta nueva regla, conceptualmente, requeriría una nueva regla y así sucesivamente, incurriéndose en un regreso al infinito. Como se advierte, este resultado no es otro que el surge de trasladar el argumento de Carroll al tema del seguimiento de reglas: planteo que ha sido célebremente formulado por Wittgenstein en los parágrafos $\$ 201$ y $\$ 202$ de sus Investigaciones Filosóficas ${ }^{7}$.

Desde ya que lo anterior no supone negar que existen criterios de corrección acerca de si una norma es o no aplicable a un caso particular. De hecho, un juez o jueza ponen en uso ese criterio cuando aplican una norma a un caso. Pero lo que no hacen es captar o comprometerse con una fórmula

6 En mi tesis doctoral (Scataglini, 2021) he analizado la cuestión de la "subsunción" en estos términos, que podrían resultar en alguna medida compatibles con el planteo de Duarte d'Almeida.

7 Para un análisis del planteo wittgensteiniano y sus consecuencias, véase Scataglini (2021, Capítulo 2), donde se establece que las conderaciones de Investigaciones Filosóficas no conducen ni al escepticismo ni a un mero convencionalismo respecto del seguimiento de reglas. En cambio, lo que el argumento wittgensteiniano muestra es que el significado de una regla no debe buscarse en nuevas reglas (i. e. "interpretaciones"). Saber seguir reglas constituye el dominio de una técnica que se adquiere mediante el entrenamiento y la incorporación en una práctica. 
Aquiles, la Tortuga y la aplicación de normas jurídicas como un "asunto...

o principio universal que contiene (extensional u intensionalmente) todas las aplicaciones de la norma.

Lo recién dicho no tiene nada que ver con adherir al particularismo jurídico. Por el contrario, de lo que estoy hablando es de en qué consiste aplicar reglas generales, las que reconozco como tales. Siguiendo a Bayón, se podría hablar de un "universalismo mínimo" respecto del seguimiento de reglas:

juzgar, en cualquier contexto no es más que clasificar un objeto particular, esto es, subsumirlo en un concepto; pero no es posible articular y explicitar ("codificar") un conjunto de reglas que fijen exhaustivamente cuándo lo particular es una instancia de lo universal, porque eso sería tanto como articular meta-reglas para la aplicación de reglas, con la consiguiente reproducción del problema en lo concerniente a la aplicación de meta-reglas (Bayón 2007, p. 150).

Bayón está aludiendo, por supuesto, al planteo wittgensteiniano antes referido, el cual constituye una crítica a la denominada visión "intelectualista” del seguimiento de reglas. En la concepción de Wittgenstein-Ryle, aplicar una regla a un caso (o dicho a la inversa, subsumir un caso bajo una regla) consiste en un saber cómo, en tener una habilidad.

Y lo importante aquí es que cuando de lo que se trata es de una habilidad, no poder explicitar cómo desarrollarla no equivale a no saber cómo desarrollarla. Podemos saber cómo hacer algo correctamente sin poder especificar en el lenguaje - y aún sin representarnos intelectualmente- de manera exhaustiva las condiciones para o el criterio de dicha corrección. ${ }^{8}$

8 Ryle critica a los defensores de la concepción intelectualista, en tanto (ellos) "reassimilate knowing how to knowing that by arguing that intelligent performance involves the observance of rules, or the application of criteria. It follows that the operation which is characterised as intelligent must be preceded by an intellectual acknowledgment of these rules or criteria; that is, the agent must first go through the internal process of avowing to himself certain propositions about what is to be done ('maxims', 'imperatives' or 'regulative propositions' as they are sometimes called); only then can he execute his performance in accordance with those dictates" (Ryle, 1949, p. 29). 
En atención a estas reflexiones podría afirmarse, para ponerlo en términos simples, que Duarte d'Almeida y yo estamos de acuerdo en qué implica - y qué no implica- subsumir un caso bajo una norma jurídica9 no implica la representación mental de, ni el compromiso con, una proposición universal que especifica todas las aplicaciones correctas de esa norma.

\section{Aplicación pragmática}

La última afirmación de la sección precedente deja abierta la cuestión de si la propuesta de Duarte d'Almeida se limita exclusivamente a la aplicación de normas jurídicas o si podría extenderse a la aplicación de cualquier regla. Por mi parte, la considero extensible, ya que entiendo que la aplicación de normas jurídicas es —más allá de sus particularidades-un caso más de la aplicación de reglas en general.

Ahora bien, una de las particularidades de la aplicación de normas jurídicas realizada por los jueces y juezas al resolver un caso individual es que se trata de una acción que tiene efectos institucionales específi$\cos { }^{10}$ Siguiendo a Austin podríamos caracterizar la aplicación del derecho

9 Dos aclaraciones terminológicas respecto de esta afirmación. La primera: estoy utilizando el término "subsumir" en el sentido del que dan cuenta mis consideraciones en el texto principal, esto es en el marco de un "universalismo mínimo"; por lo tanto considero que, así entendido, determinar que un caso "se subsume" bajo una norma es equivalente a reconocer que la norma se aplica a ese caso. La segunda, al utilizar "caso" me refiero aquí a lo que Alchourrón y Bulygin (1971 [2002], pp. 58-59) han distinguido como "caso individual", en contraposición a "caso genérico". Esta conocida distinción de los profesores argentinos aparece en parte recogida por Duarte d'Almeida en la última parte de su trabajo, aunque con algunas diferencias de alcance y especificidad por parte del autor (ver Duarte d'Almeida, 2021, Sección 5). Con todo es dable destacar que la subsunción de un "caso individual" bajo una norma podría ser expresada también en términos de que un caso genérico (aquel que mediante el uso de predicados recoge los rasgos caracterísiticos del caso individual) queda subsumido bajo la norma; a ello se alude como "subsunción genérica".

10 Afirma Duarte d'Almeida: "No es en nuestra calidad de ciudadanas y ciudadanos que aplicamos pragmáticamente disposiciones jurídicas. Solo aplicamos pragmáticamente una disposición jurídica cuando, en alguna función o calidad específica — como es, paradigmáticamente, la de un juez- ejercemos (o al menos pretendemos ejercer) la autoridad para llevar una determinada cuestión o controversia a una resolución de algún tipo” (2021, Sección 3.5) 
Aquiles, la Tortuga y la aplicación de normas jurídicas como un "asunto...

mediante una sentencia judicial como un acto de habla performativo. ${ }^{11} \mathrm{Al}$ pronunciar o firmar una sentencia los jueces y juezas hacen algo: absuelven, condenan, anulan cláusulas contractuales, ordenan llevar adelante una ejecución, etc.

Pero este aspecto - llamémoslo "performativo" - no parece ser el único al que Duarte d’Almeida alude como "aplicación pragmática" del derecho. El autor involucra bajo esta idea otras dos cuestiones: i) la evaluación por parte del juez o jueza de que no hay consideraciones compensatorias para llegar a la conclusión, y ii) que su decisión está jurídicamente justificada por el derecho existente.

Al referirse a la relación entre esta "aplicación pragmática" y la "aplicación inferencial” del derecho señala:

la justificación de los enunciados de aplicabilidad (y de las afirmaciones sobre sus consecuencias) se basa en una serie de consideraciones sustantivas, a las cuales un tribunal debe ser sensible. Entre ellas figuran las consecuencias adicionales que se derivarán (o derivarían) si la conclusión que resulta de la aplicación inferencial de esa disposición realmente se considera verdadera en términos jurídicos, y si se actúa en consecuencia (Duarte d'Almeida, 2021, Sección 4).

Y también:

(...) el hecho de que haya una determinada decisión $d$ que el tribunal deba jurídicamente emitir $s i$ una disposición $p$ se aplica con una determinada consecuencia a un objeto $x$ es en sí mismo una consideración que debe tener en cuenta el tribunal al determinar si $p$ se aplica o no a $x$. Si $d$ es una decisión sumamente indeseable o absurda, entonces esa es una consideración que en sí misma incide en el razonamiento del tribunal sobre si se aplica o no la disposición pertinente. La aplicación pragmática del derecho, para decirlo

${ }^{11}$ John Austin (1962) caracteriza a los performatives como aquellos verbos o enunciados que los contienen tales que el acto de expresar la oración es realizar (to perform) una acción. En la versión en castellano los traductores utilizan el término "realizativos" (1962 [1971], p. 45). Aquí prefiero usar "performativos". 
de modo sintético, retroalimenta normativamente la aplicación inferencial del derecho. (Duarte d’Almeida 2021, Sección 4)

No estoy segura de que la clasificación en aplicación inferencial y aplicación pragmática tal como la presenta en su trabajo Duarte d’Almeida resulte iluminadora.

$\mathrm{Si}$, como he sugerido, inferir se entiende como un saber hacer, evaluaciones sustantivas como por ejemplo si existen consideraciones compensatorias a tener en cuenta, podrían contar como parte de la aplicación inferencial. Duarte d'Almeida parece reconocer esto en el inicio de la primera cita supra. Pero al mismo tiempo, en su planteo general, parecería asumir que la evaluación de las consideraciones compensatorias corresponde exclusivamente a la aplicación pragmática, por ejemplo en la segunda cita supra cuando alude a aquellas consideraciones que podrían llevar a rechazar un resultado absurdo, como una muestra de que la aplicación pragmática retroalimenta normativamente a la aplicación inferencial.

Pero lo cierto es que el resto de los ciudadanos y ciudadanas que no somos jueces ni juezas también realizamos inferencias que involucran los dos aspectos que Duarte d'Almeida clasifica como correspondientes a la "aplicación pragmática", y que identifiqué antes como i): la evaluación de si hay o no consideraciones compensatorias para arribar a la conclusión, y ii): la de que la conclusión está jurídicamente justificada de acuerdo al derecho vigente. Esto es precisamente lo que hacemos, por ejemplo, abogados y abogadas litigantes y también juristas o quienes se dedican a la dogmática: evaluamos posibles consideraciones compensatorias y sostenemos nuestras conclusiones como ajustadas a derecho, como la determinación correcta de lo que el derecho dispone. Solo que nuestros argumentos y conclusiones no constituyen actos de habla performativos: carecen de consecuencias institucionales.

Por esta razón, me parece que resulta más claro reservar el rótulo de "aplicación pragmática" para dar cuenta del aspecto distintivo de la aplicación del derecho por parte de jueces y juezas: lo que he llamado el aspecto "performativo" o institucional; los jueces y juezas "aplican pragmáticamente" el derecho porque lo hacen en el marco de una institución -la práctica jurídica - que les otorga autoridad para resolver conflictos con- 
Aquiles, la Tortuga y la aplicación de normas jurídicas como un "asunto...

forme a las normas vigentes; autoridad de la que carecen el resto de las personas, incluyendo abogados y abogadas.

A diferencia de Duarte d'Almeida, creo que los otros dos aspectos que él incluye en la "aplicación pragmática" no resultan exclusivos de la decisión judicial. Ya que si la acción de inferir se concibe como una actividad inteligente -i.e. un saber hacer en el marco de una práctica - la misma incluye consideraciones sustantivas relativas a cuál es la solución "jurídicamente justificada" o "conforme a derecho", también cuando recae en algunos otros participantes de la práctica jurídica que no son jueces. Pensándolo bien, quizás la propuesta que mejor recoge el espíritu ryleano que he seguido, sea la de atribuir el título "aplicación pragmática" a estas inferencias inteligentes y acuñar "aplicación performativa” para el resultado específico del quehacer de los jueces.

\section{Bibliografía}

Alchourrón, C. y Bulygin, E. (1971). Normative Systems. Viena: SpringerVerlag. Versión en castellano: Introducción a la metodología de las ciencias jurídicas y sociales. Buenos Aires:Astrea, 2002.

Austin, J. L. (1962). How to do Things with Words. Oxford: Oxford University Press. Versión en castellano: Cómo hacer cosas con palabras, tr. G. Carrió y E. Rabossi. Buenos Aires: Paidós, 1971.

Bayón, J. C. (2007). Bulygin y la justificación de las decisiones judiciales: la parte sorprendente. En J.J. Moreso y C. Redondo, Un diálogo con la teoría del derecho de Eugenio Bulygin (pp. 137-152). Barcelona - Madrid: Marcial Pons.

Brandom, R. (2000). Articulating reasons. An introduction to Inferentialism. Harvard: Harvard University Press. Edición en castellano: La articulación de las razones. Una introducción al inferencialismo. Madrid: Siglo XXI de España, 2002.

Carroll, L. (1895). What the Tortoise Said to Achilles, Mind, 4(14), 278-280 Duarte d'Almeida, L. (2019). On the legal syllogism. En D. Plunkett, S. Shapiro y K. Toh (eds.), Dimensions of Normativity: New Essays 
on Metaethics and Jurisprudence (pp. 335-364). New York: Oxford University Press.

Duarte d'Almeida, L. (2021). ¿Qué es aplicar el derecho? Discusiones, 27. Hart, H. L. A. (1963). El Concepto de Derecho. Buenos Aires: Abeledo Perrot.

Ryle, G. (1949). The concept of mind. London: Hutchinson's University Library. 2008.

Scataglini, M. G. (2021). Seguimiento de reglas: el aguijón pragmático en la teoría del derecho. Barcelona-Madrid: Marcial Pons.

Winch, P. (1958). The Idea of a Social Science and its Relation to Philosophy. London: Routledge, Second edition. 1990.

Wittgenstein, L. (1953). Investigaciones Filosóficas. México: Instituto de Investigaciones Filosóficas de la UNAM. 1988. 